\title{
Characterization and toxicity of citral incorporated with nanostructured lipid carrier
}

\author{
Noraini Nordin ${ }^{1}$, Swee Keong Yeap ${ }^{2,3}$ ， Nur Rizi Zamberi ${ }^{1}$, Nadiah Abu ${ }^{1,4}$ ， Nurul Elyani Mohamad ${ }^{1}$, Heshu \\ Sulaiman Rahman ${ }^{5}$, Chee Wun How ${ }^{3,6}$, Mas Jaffri Masarudin ${ }^{1,3}$, Rasedee Abdullah ${ }^{3}$, Noorjahan Banu \\ Alitheen Corresp. 1,3 \\ ${ }^{1}$ Department of Cell and Molecular Biology, Faculty of Biotechnology and Biomolecular Sciences, Universiti Putra Malaysia, Serdang, Selangor, Malaysia \\ 2 China-ASEAN College of Marine Sciences, , Xiamen University Malaysia, Sepang, Malaysia \\ 3 Institute of Bioscience, Universiti Putra Malaysia, Serdang, Selangor, Malaysia \\ 4 UKM Medical Centre, UKM Medical Molecular Biology Institute (UMBI), Cheras, Wilayah Persekutuan, Malaysia \\ 5 Department of Microbiology, Faculty of Veterinary Medicine, University of Sulaimanyah, Sulaimanyah, Kurdistan Region, Iraq \\ 6 Faculty of Pharmacy, MAHSA University, Jenjarom, Malaysia \\ Corresponding Author: Noorjahan Banu Alitheen \\ Email address: noorjahan@upm.edu.my
}

Nanoparticle as a cancer drug delivery vehicle is rapidly under investigation due to its promising applicability as a novel drug delivery system for anticancer agents. This study describes the development, characterization and toxicity studies of a nanostructured lipid carrier (NLC) system for citral. Citral was loaded into the NLC using high pressure homogenization methods. The characterizations of NLC-citral were then determined through various methods. Based on Transmission Electron Microscope (TEM) analysis, NLCCitral showed a spherical shape with an average diameter size of $54.12 \pm 0.30 \mathrm{~nm}$ and a polydipersity index of $0.224 \pm 0.005$. The zeta potential of NLC-Citral was $-12.73 \pm 0.34$ $\mathrm{mV}$ with an entrapment efficiency of $98.9 \pm 0.124 \%$, and drug loading of $9.84 \pm 0.041 \%$. Safety profile of the formulation was examined via in vitro and in vivo routes to study its effects toward normal cells. NLC-Citral exhibited no toxic effects towards the proliferation of mice splenocytes. Moreover, no mortality and toxic signs were observed in the treated groups after 28 days of treatment. There were also no significant alterations in serum biochemical analysis for all treatments. Increase in immunomodulatory effects of treated NLC-Citral and Citral groups was verified from the increase in CD4/CD3 and CD8/CD3 T cell population in both NLC-citral and citral treated splenocytes. This study suggests that NLC is a promising drug delivery system for citral as it has the potential in sustaining drug release without inducing any toxicity. 
Noraini Nordin ${ }^{1}$, Yeap Swee Keong ${ }^{2,3}$, Nur Rizi Zamberi ${ }^{1}$, Nadiah Abu ${ }^{1,4}$, Nurul Elyani Mohamad ${ }^{1}$, Heshu Sulaiman Rahman ${ }^{5}$, Chee Wun How ${ }^{2,6}$, Mas Jaffri Masarudinn ${ }^{1,2}$, Abdullah Rasedee ${ }^{2}$, Noorjahan Banu Alitheen ${ }^{1,2}$

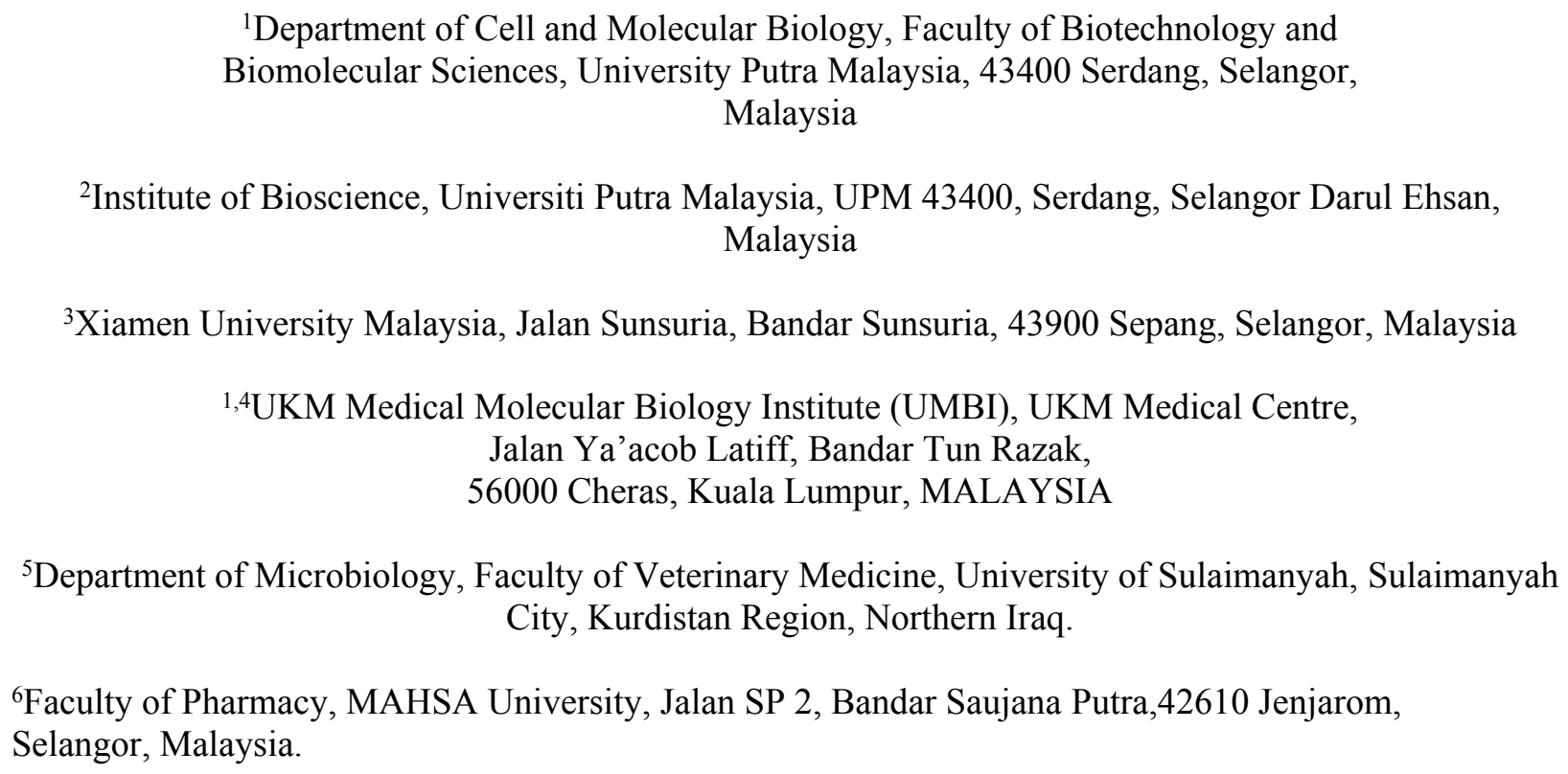

Corresponding author: Noorjahan Banu Alitheen ${ }^{1}$

E-mail: [noorjahan@upm.edu.my] 


\section{Abstract}

36 Nanoparticle as a cancer drug delivery vehicle is rapidly under investigation due to its promising

37 applicability as a novel drug delivery system for anticancer agents. This study describes the development, 38 characterization and toxicity studies of a nanostructured lipid carrier (NLC) system for citral. Citral was

39 loaded into the NLC using high pressure homogenization methods. The characterizations of NLC-citral 40 were then determined through various methods. Based on Transmission Electron Microscope (TEM) 41 analysis, NLC-Citral showed a spherical shape with an average diameter size of $54.12 \pm 0.30 \mathrm{~nm}$ and a 42 polydipersity index of $0.224 \pm 0.005$. The zeta potential of NLC-Citral was $-12.73 \pm 0.34 \mathrm{mV}$ with an 43 entrapment efficiency of $98.9 \pm 0.124 \%$, and drug loading of $9.84 \pm 0.041 \%$.NLC-Citralexhibited no toxic 44 effects towards the proliferation of mice splenocytes. Moreover, no mortality and toxic signs were 45 observed in the treated groups after 28 days of treatment. There were also no significant alterations in 46 serum biochemical analysis for all treatments. Increase in immunomodulatory effects of treated NLC-

47 Citral and Citral groups was verified from the increase in CD4/CD3 and CD8/CD3 $\mathrm{T}$ cell population in 48 both NLC-citral and citral treated splenocytes. This study suggests that NLC is a promising drug delivery 49 system for citral as it has the potential in sustaining drug release without inducing any toxicity. 


\section{Introduction}

57 Exploring natural plant products as an avenue to discover novel chemical entities is one of the 58 fastest growing areas of pharmaceutical research, considering that bioactive agents are pivotal as

59 potential cures for various diseases. There have been more than one thousand plants identified to 60 possess significant anticancer properties (Cragg \& Newman, 2005). Citral, with the molecular

61 formula $\mathrm{C}_{10} \mathrm{H}_{16} \mathrm{O}$ is an aldehyde that is widely used in perfumes for its citrus scent. Citral has

62 been claimed to possess anti-inflammatory effects, as it inhibited NO production and suppresses

63 the activation of NF-kappa B in RAW264.7 cells (Jeong et al., 2008). Treatment with citral also

64 decreased cell proliferation and altered the mitochondrial membrane potential resulting in 65 apoptotic induction of HeLa and ME-180 cervical cancer cell lines (Ghosh, 2013). Together, 66 these versatile characteristics of citral have attracted researchers to further exploit its increased 67 efficiency. However, despite its multi-beneficial effects citral remains an unstable molecule in 68 acidic environments and non-soluble in water (Weerawatanakorn et al., 2015). Therefore, the 69 incorporation of citral into colloidal dispersions such as nano-emulsions in particular, could be 70 the best way to stabilize the poorly-soluble compound.

72 To increase the body fluid saturation solubility of poorly soluble compounds, a new delivery 73 system needs to be developed. The interest of using nanoparticles as a drug delivery system is 74 aimed to improve the bioavailability of drugs, increase the release time in the human body, 75 targeting the drug to its site of action to introduce its physiochemical stability of the drug 76 (Mudshinge, Deore, Patil, \& Bhalgat, 2011). Different types of nanocarriers, including lipid 77 nanoparticles have been developed over time for this purpose. Lipid nanoparticles have many 
78 desirable features which includes low toxicity, a biodegradable matrix, high capacity to

79 incorporate lipophilic and hydrophilic drugs, and controlled release properties of incorporated

80 drugs (Kasongoet al., 2011). The nanostructured lipid carrier (NLC), a second generation of Solid

81 Lipid nanoparticle (SLN)confers all these properties and holds great promise to become an

82 excellent drug carrier system (Pardeshi et al., 2012). NLC has greater flexibility for drug loading

83 and exhibits a reduced drug expulsion of the formulation over time that contributes towards long

84 term stability and better modulation of drug release (Almeidaet al., 2014) as compared to SLN.

85 NLCs are considered as one of the most versatile generation of lipid nanoparticle as oral drug 86 delivery systems to treat cancer. Studies has reported that small particles of NLCs possessing a

87 particle size less than $200 \mathrm{~nm}$ have reduced uptake by the Kupffer cells in the liver and 88 prolonged circulation time in blood, allowing better uptake by tumors (Montenegro et al., 2011).

89 Additionally, NLC appears to be an attractive approach for the delivery of lipophilic cancer 90 drugs such as Tamoxifen and 5-Fluorouracil (Andalib et al., 2012; How et al., 2013). With NLC, 91 the drug is expected to solubilize into the hydrophobicphase incorporated into the core of the 92 solid lipid. This could enhance the loading capacity, entrapment efficiency and also control the 93 drug release from the NLC in time. Thus far, there exist no reports which disclose the use of 94 nanostructured lipid carrier as an efficient carrier system to increase the solubility of citral and 95 the physicochemical properties.

96 One of the major concerns with a nanoparticle system is its inherent toxicity. The safety profiles 97 of citral has been studied on $\mathrm{F} 344 / \mathrm{N}$ rat and $\mathrm{B} 6 \mathrm{C} 3 \mathrm{~F} 1$ mice for two years and been concluded as a 98 non-carcinogenic compound (Ress et al., 2003). The potential success of a nanoparticle system 99 relies on many factors, with one of it the minimum toxicity effects exhibited by the carrier itself.

100 Studies has reported that NLC induces no toxicity and no mortality in an acute toxicity study 
101 using BALB/c mice (Rahman et al., 2014). Besides the extensive applicability of lipid-based

102 nanoparticle, the toxicity of this system has not been adequately studied thus far. In this present

103 study, combination of hydrogenated palm oil as a solid lipid whilst olive oil a liquid lipid was

104 chosen because this combination was tested to be the least cytotoxic on normal BALB/C mice 105 and 3 T3 cells (How, Rasedee, \& Abbasalipourkabir, 2013).

106 As a potential drug carrier, it is essential to examine the safety and toxicity of citral incorporated

107 into the NLC system to ensure its suitability for the future therapeutic usage of the formulation.

108 For these reasons, the aim of this present work was to determine the physicochemical properties 109 of NLC-citral and to assess the toxicological effects in BALB/c mice treated with NLC-citral.

\section{Materials and Method}

112

113 The materials used to synthesize the Nanostructured Lipid Carrier in this project were received

114 from the Institute of Bioscience, UPM without any alteration in the processing method.

115 Hydrogenated palm oil (HPO) was a gift from Institute of Bioscience (Selangor, Malaysia),

116 Lipoid S-100 (Merck Millipore, Germany), and Olive oil (Basso, Italy). Thimerosal, D-Sorbitol,

117 Tween-80, citral 95\%, Dulbecco's Modified Eagle's Medium (DMEM), fetal bovine serum

118 (FBS), Thiazolyl Blue Tetrazolium Bromide were all purchased from Sigma-Aldrich (MO,

119 USA). All of the reagents used were of analytical grade except for olive oil. 


\section{Preparation of nanostructured lipid carriers and loading of Citral}

122

123 The NLCs were prepared using a high-pressure homogenization technique. The synthesis method

124 of NLC loaded with citral was similar to a previous study with slight modifications (Rahman et

125 al., 2013a). The lipid phase was composed of hydrogenated palm oil (HPO), Lipoid S-100 and

126 Olive oil at a ratio of 7:3:3 that were mixed in a beaker and heated at $70^{\circ} \mathrm{C}$. Five hundred $\mathrm{mg}$ of

127 citral was added into the lipid fraction with constant stirring (1000 rpm) for 5 minutes. Complete

128 yellowish in color mixture of drug was ensured before proceeding with the next step. For

129 aqueous phase, D-Sorbitol (4.75 \% [w/v]), Thimerosal $(0.005 \%[\mathrm{w} / \mathrm{v}])$, and 1\% Tween-80 were

130 dissolved in double-distilled water at $70^{\circ} \mathrm{C}$ and was then added into the lipid phase under

131 constant stirring for 5 minutes. Further mixing was done by using a high speed stirring method

132 with Ultra-Turrax ${ }^{\circledR}(\mathrm{IKA} /$ Staufen, Germany) at 13,000 rpm for 10 minutes. The pre-emulsion

133 mixture was then pressurized using a high pressure homogenizer (Avestin, Canada) at 1000 bar

134 for 15 cycles at $70^{\circ} \mathrm{C}$. Finally the hot and clear nanoemulsion was sealed and allowed to cool

135 down at room temperature $\left(27^{\circ} \mathrm{C}\right)$ for 24 hours to recrystallize and form the NLCs. The blank

136 NLC was formulated exactly with the same method but without the addition of citral.

\section{Zeta potential (ZP)}

139 The magnitude charges between the particles in the NLC were determined by using Zetasizer

140 Nano ZS (Malver, Germany). NLC was diluted 1:1 ratio with double distilled water

141 appropriately prior to measurements to get optimum of 50-200 kilo counts per second 142 (Thatipamula et al., 2011). 


\section{Particle size}

145 The average particle size diameter and polydispersity index (PDI) of the NLC were analyzed by 146 using dynamic light scattering (DLS) integrated in a Zetasizer Nano ZS (Malvern Instrument,

147 Germany). The NLC solution was diluted at 1:1 ratio with double distilled water preceding the

148 measurement. Five independent measurements were obtained at $25^{\circ} \mathrm{C}$ with particle size and PDI 149 (size distribution) were calculated.

\section{Transmission electron microscopy (TEM)}

152

153 The NLC-Citral sample was dropped slowly on the surface of a copper grid coated with carbon

154 and then let to air drying for about 5 minutes. Negative staining was performed by using $1 \%$ 155 phosphotungstic acid then allowed for air drying for another 3 minutes. The sample was then 156 visualized with transmission electron microscopy (Hitachi H-7100, Japan).

\section{Entrapment efficiency (EE) and drug loading (DL) capacity}

160 The amount of free drug in the sample was measured in order to determine the entrapment

161 efficiency and the drug loading capacity of the sample by using ultrafiltration method. This was

162 performed using Centisart filter tubes (Sartorious, Germany) with molecular weight cut off $163300 \mathrm{kDa}$. Five $\mathrm{mL}$ of NLC-citral was placed in the upper chamber of the tubes and then was 
164 centrifuged using Eppendorf Centrifuge (Hamburg, Germany) at $15000 \mathrm{rpm}$ for 15 minutes. The

165 foundation behind this technique is that citral filtered out to the bottom chamber is unbound to

166 the NLC while the citral remained in the top chamber were still bound as NLC-citral.

167 Subsequently, citral in the bottom chamber was quantified using UV-Vis spectrophotometer at

$168280 \mathrm{~nm}$ (Beckton Coulter, USA). The EE and DL percentage of the sample were calculated

169 based on the method previously reported (Rahman et al., 2013).

170

171

$$
E E(\%)=\frac{(\text { Total amount of citral })-(\text { Free amount of citral })}{(\text { Total amount of citral })} \times 100
$$

$$
D L(\%)=\frac{(\text { Total amount of citral encapsulated into NLC })}{(\text { Total amount of lipid used in NLC - citral formulation })} \times 100
$$

\section{In vitro drug release study}

175

176 The in vitro drug release study of NLC-citral was accomplished using a Franz diffusion cell

177 system (PermeGear, USA) with a receptor volume of $20.1 \mathrm{~cm}^{3}$ and effective diffusion area of

$1784.9 \mathrm{~cm}^{2}$. This study is essential to determine the suitability of the drug in the system and to check

179 the quality of the formulation. There were donor and receptor compartments in the system with

180 donor cell having the surface area of $20.1 \mathrm{~cm}^{2}$. The release of the NLC-citral was studied for 48

181 hours. Firstly, the synthetic cellulose acetate membrane (Advantec, Japan) with pore size of 200

$182 \mathrm{~nm}$ was soaked in the receiving media for 2 hours. Next, the membrane was inserted onto the

183 system and $500 \mu \mathrm{L}$ of NLC-citral suspension were dispensed in the donor compartment (Salerno 
184 et al.,2010). The receptor compartment was filled with the receiving medium (PBS $+2 \%$ SDS) at

$185 \mathrm{pH}$ 7.4. Throughout the study, the temperature of the system was maintained at $37^{\circ} \mathrm{C}$ under

186 constant stirring. At certain time intervals, $500 \mu \mathrm{L}$ of sample was withdrawn from the medium

187 in receptor compartment by using a $3 \mathrm{~mm}^{3}$ syringe needle and then refilled back by using the

188 fresh receiving medium. The samples were then analyzed by UV-Vis spectrophotometer at 280

$189 \mathrm{~nm}$ (Beckman Coulter, USA) to determine the amount of citral released from the NLC. The

190 concentration of drug release was calculated based on the standard curve for pure citral ranging

191 from $100 \mu \mathrm{g} / \mathrm{mL}$ to $10 \mu \mathrm{g} / \mathrm{mL}$. The experiment was conducted in triplicate and the drug release

192 data were evaluated by zero-order, first-order, and Higuchi equations to determine the release

193 kinetics (Rahman et al., 2013).

194

195 In vitro splenocyte viability (MTT)

196

197 In brief, upon mice sacrifice, the spleen collected was washed with $5 \mathrm{~mL}$ of PBS. It was then

198 lysed with $2 \mathrm{~mL}$ lysis buffer (155 mMNH${ }_{4} \mathrm{Cl}, 0.1 \mathrm{M} \mathrm{Na}_{2} \mathrm{EDTA}, 12 \mathrm{mM} \mathrm{KH}_{2} \mathrm{PO}_{4}$ at $\mathrm{pH}$ 7.4) for

19910 minutes to remove the red blood cells. Next, the splenocytes were seeded at a density of $2002 \times 10^{6}$ cells $/ \mathrm{mL}$ in a $96-$ well plate. Cells were treated with seven different concentrations: NLC201 Blank, NLC-citral and citral starting from $0.8 \mu \mathrm{g} / \mathrm{mL}$ to $30 \mu \mathrm{g} / \mathrm{mL}$ for 24 hours of incubation in a $20237^{\circ} \mathrm{C}$ incubator with $5 \% \mathrm{CO}_{2}$ (Memmert, USA). Then, $20 \mu \mathrm{L}$ of MTT3-[4,5-dimethylthiazol-2203 yl]-2,5 diphenyltetrazolium bromide $(5 \mathrm{mg} / \mathrm{mL})$ (Merck, USA) solution was added into the cells 204 and incubated for another 4 hours in the incubator. The plate was spun down at $1500 \mathrm{x} \mathrm{g}$ for 5 205 minutes. Next, the supernatant was removed and $100 \mu \mathrm{L}$ of dimethyl sulfoxide (DMSO) (Fisher 
206 Scientific, USA) was added in to the plate and incubated for another 15 minutes at room

207 temperature. The plate was read with microplate reader at $570 \mathrm{~nm}$. In vitro splenocytes viability 208 assay was done according to the previous study (Abu et al., 2015). The percentage of cells 209 viability was calculated based on the formula below:

$$
\text { Viability }(\%)=\frac{\text { OD of sample }}{\text { OD of control }} \times 100
$$

\section{Animal}

215 Male BALB/c male mice aged seven weeks (20-22 g) were purchased from the Animal House of

216 Faculty of Veterinary, Universiti Putra Malaysia (UPM, Malaysia). This study was approved by 217 the Institutional Animal Care and Use Committee, Universiti Putra Malaysia (R098/2014). The 218 mice were randomly selected and grouped into 3 groups with five mice per group; NLC-Blank,

219 NLC-citral and citral. Fifteen mice were acclimatized in the laboratory environment for 7 days.

220 The mice were accommodated in polypropylene plastic cage with pellet and water provided ad 221 libitum during the period of study. The treatment of $50 \mathrm{mg} / \mathrm{kg} /$ day of NLC-citral, citral and 222 NLC-Blank were administered orally for 28 days following the method described earlier 223 (Mohamad et al., 2015). The animals were observed for any toxic signs such as loss of weight, 224 shedding of fur or behavioral abnormalities after 28 days of study. 


\section{Serum Biochemistry}

227

228 Blood samples were collected in a plain tube from the sacrificed mice by cardiac puncture. The

229 serum was obtained by centrifugation at $3000 \mathrm{rpm}$ for 15 minutes. The concentration of aspartate

230 aminotransferase (AST), alanine aminotransferase (ALT), alkaline phosphatase (ALP) and

231 creatinine level in mice serum were analyzed accordingly using Hitachi automatic analyzer

232 (Hitachi-902, LTD, JAPAN).

234 In vivo Immunophenotyping of Splenocytes

235

236 Mice spleen obtained from the sacrificed mice were washed with Phosphate Buffered Saline

237 (PBS) and meshed through a $40 \mu \mathrm{m}$ sterile filter with plunger. Next, the suspension was washed 238 with PBS and centrifuged at $2000 \mathrm{x}$ g for 15 minutes to get the cells pellet. Then, the red blood 239 cell was lysed with $2 \mathrm{~mL}$ of lysis buffer $\left(155 \mathrm{mMNH}_{4} \mathrm{Cl}, 0.1 \mathrm{M} \mathrm{Na} 2 \mathrm{EDTA}, 12 \mathrm{mM} \mathrm{KH}_{2} \mathrm{PO}_{4}\right.$ at

$240 \mathrm{pH} 7.4$ ) and incubated for 10 minutes in $4^{\circ} \mathrm{C}$.After that, the suspension was again spun at $2000 \mathrm{x}$

$241 \mathrm{~g}$ for 5 minutes. Cell was resuspended with PBS for counting purposes. About $1 \times 10^{6}$ cells $/ \mathrm{mL}$ 242 was stained with antibodies (CD3, CD4, CD8) (Abcam, USA) for 2 hours with constant shaking.

243 Then, the cells were fixed in $600 \mu \mathrm{L}$ of $4 \%$ paraformaldehyde (Sigma, USA) before being 244 subjected to flow cytometry analysis (FACS Calibur, BD, USA). 


\section{In vivo Nitric Oxide detection of the spleen}

249 Nitric Oxide detection from the spleen was established with the Griess Reagent Kit (Sigma, 250 USA). The experiment was conducted according to the protocol provided with the kit. The 251 supernatant from the splenocytes was mixed with Griess Reagent and incubated for 30 minutes

252 before being measured at $548 \mathrm{~nm}$ using microplate reader (Beckman Coulter, USA).

254

255

\section{Results and discussion}

\section{Physicochemical Characterisation of NLC-Citral formulation}

\section{Particle size and Polydispersity Index}

262 NLC-Citral was successfully synthesized using the high pressure homogenization method. The 263 summary of physicochemical characteristics of NLC-citral formulation is as shown in Table 1.

264 Particle size is the most fundamental parameter in a nanoparticle study. The particle size and 265 polydispersity index is highly important in determining the physical stability of colloidal 
266 dispersion system of the nanoparticle (Lee et al., 2007). The mean particle size and

267 polydispersity index of NLC-citral were obtained as $54.12 \pm 0.30 \mathrm{~nm}$ and $0.224 \pm 0.005$, 268 respectively.

269 Particle size of the nanoparticle was present in the average diameter of the nanoparticle. It is 270 crucial to confirm that the size of the nanoparticle formulated is within nano-sized range 271 (Mukherjee et al., 2008). As depicted in Figure 1, NLC-citral was observed to confer regular 272 shapes and are relatively uniform suggesting good particle dispersity. There was a minor 273 difference in size distribution data obtained from TEM and zeta sizer. This small disparity in 274 measurement was probably due to differences in the techniques and method of data analysis 275 between both instruments. The measurement of nanoparticles is mostly influenced by the 276 conditions, method of analyzing data and also depends on the operation of instrument in the 277 determination (How et al., 2013). From this result, it was concluded that this formulation has 278 fallen within a nano-sized range. The size of the particle plays a key role towards their adhesion 279 to and interaction with biological cells (Foster et al.,2001). Being a small nanoparticle, NLC280 Citral holds a potential in lowering the risk of non-specific liver uptake (Seki et al., 2004) as well 281 as increasing the actual distribution and bioavailability of the $\operatorname{drug}($ Fang et al., 2006). The 282 incorporation of a water insoluble anticancer compound (Amoitone B) with NLC was reported to 283 enhance its bioavailability (Luan et al., 2013). Moreover, small nanoparticle with a diameter size 284 of $50-60 \mathrm{~nm}$ resulted in high tumor uptake as well as sustained release of the drug in cancer 285 biology studies (Mitra et al., 2001; Sharma et al., 1996). 
288 Table 1: The summary of characterizations for NLC-Citral

\begin{tabular}{cc}
\hline Element & Value \\
\hline Zeta potential & $-12.59 \pm 0.52 \mathrm{mV}$ \\
Zeta sizer & $54.12 \pm 1.10 \mathrm{~nm}$ \\
Polydispersity index & $0.224 \pm 0.005$ \\
Entrapment Efficiency & $98.9 \pm 0.124 \%$ \\
Drug Loading Capacity & $9.84 \pm 0.041 \%$ \\
\hline
\end{tabular}

289

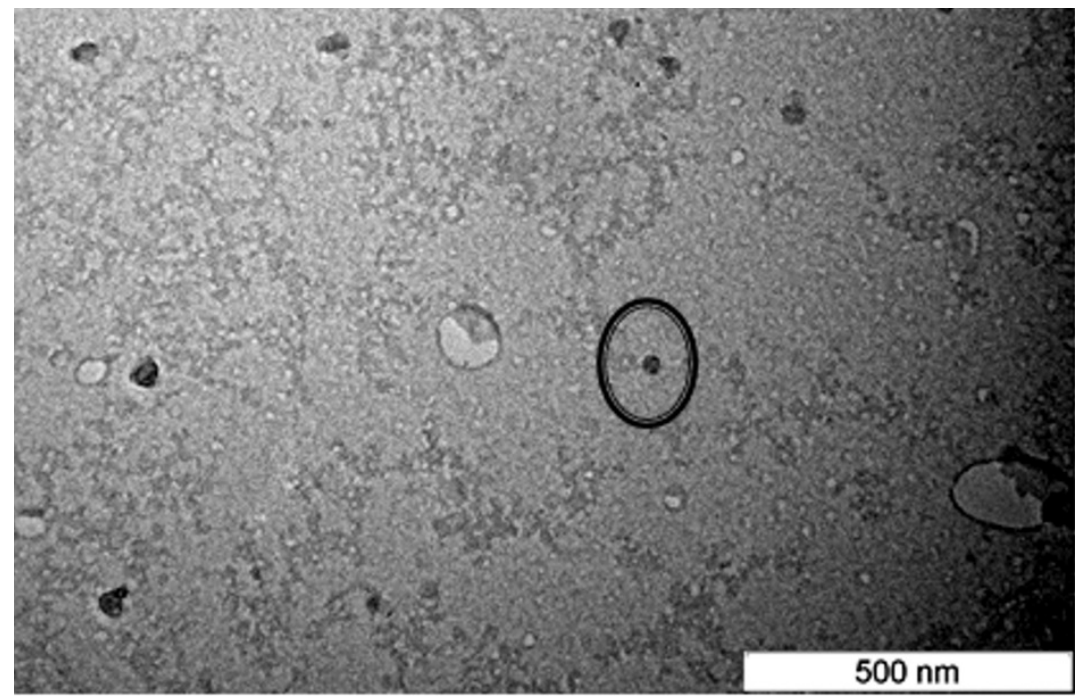

291 Figure 1: Transmission electron microscope (TEM) image of the NLC-Citral prepared by 292 negative staining at $\mathbf{8 0 , 0 0 0 X}$ magnification. The circle represents the nanoparticle which 293 can be seen in regular circle shape.

296 Determination of zeta potential for NLC-citral was done to evaluate the overall stability of the

297 formulation. The average zeta potential for NLC-citral was $-12.73 \pm 0.34 \mathrm{mV}$ (Table 1). Stability 
298 test for a nano-suspension is very crucial in any nanoparticle studies. Zeta potential is one of the

299 parameters that govern the stability of a nanoparticle formulation. As a rule of thumb, zeta

300 potential ranging from $-5 \mathrm{mV}$ to $+5 \mathrm{mV}$ indicates low stability and higher possibility for

301 aggregation (Honary \& Zahir, 2013). Generally, a zeta potential value must be higher than

$302+30 \mathrm{mV}$ or lower than $-30 \mathrm{mV}$ (Rahman et al., 2013a). Surfactants were used as a steric stabilizer

303 during the nanoparticle formulation to control particle size and the stability of dispersion.

304 Surfactants have a wide range of uses in pharmaceutical preparations. In this formulation, Tween

30580 was chosen to provide a good steric stabilization. Previous finding has proved that Tween 80

306 can compensate the stability of NLC and SLN dispersions by providing a steric stability in

307 Domeperidone loaded with NLC and SLN (Thatipamula et al., 2011).Conversely, studies have

308 concluded that, nanosuspension with slightly low zeta potential is stable enough with the

309 additional usage of higher molecular weight of stabilizer which will act by steric stabilization in

310 the particle (Honary \& Zahir, 2013; Lee et al., 2007).Hence, the stabilizer used in the

311 formulation of nanoparticle effected the average size and charges of the NLC (Abdelwahab et 312 al., 2013).

\section{Entrapment Efficiency and Drug Loading Capacity}

315 The entrapment efficiency study is required to determine the high performance from the

316 formulation. In this formulation of NLC, Citral conveyed $98.9 \pm 0.124 \%$ of entrapment

317 efficiency in the system with $9.84 \pm 0.041 \%$ of drug loading capacity in the lipids concentration.

318 Incorporation of citral has led to a high percentage of entrapment efficiency (EE), probably

319 because of its lipophilicity and low water solubility characters. High EE is required for good 
320 encapsulation parameters in any drug nano-carrier system including NLC. Furthermore, the

321 amount of drug entrapped in the nano-carrier also determines the performance of drug delivery

322 system since it influences the rate of the drug release from the system (Tiwari et al., 2012). The

323 addition of drugs in the NLC system minimizes the interfacial retention in between lipid matrix

324 and liquid phase whereby reduces the free energy within phase boundary of lipid and the drug

325 incorporated (How et al., 2013). Ideally a high loading capacity is aimed because it would

326 improve the entrapment efficiency of the drug eventually (Patel et al., 2012). Drug loading is

327 defined as the process of incorporation of the drug into the carrier system; meanwhile entrapment

328 efficiency portrays the effectiveness of the incorporated drug into the carrier. The solubility of

329 the drug in lipid used assures the maximum drug loading in the NLC system (Rahman et al.,

330 2013a).Hence, high loading capacity of the citral in NLC system examined was due to the good

331 solubility of citral in the lipid phase of the NLC. The formation of unordered lipid crystal in the

332 NLC has increased the loading capacity. Imperfect crystal formation in the lipid nanoparticle can

333 be achieved through the mixing of solid lipid with liquid lipid which would lead to a higher drug

334 loading of NLC (Müller, Radtke, \& Wissing, 2002).

\section{Drug Release Study}

337 Furthermore, the drug release profile of NLC-citral was also determined in this study. The

338 cumulative percentage of citral from NLC-citral over 48 hours is as shown in Figure 2. In vitro

339 drug release study was performed using the Franz diffusion cell system in order to determine the

340 release profile of the citral from NLC-citral for 48 hours. NLC system has shown a constant

341 release rate of citral release with a total of $96.3 \pm 2.1 \%$ after 48 hours of study. 
342 It has become an essential strategy that drug delivery system should possess a better drug release

343 capacity to improve its effectiveness and reduce side effects of the drug caused by a rapid dosage

344 consumption by the patients in a therapeutic routine(How et al., 2013).It was observed that a

345 slow release profile of citral from NLC was apparent, compared to the administration of citral

346 alone (Figure 2). This study also showed that the drug release kinetics of citral from NLC

347 followed a zero-order kinetic model, with an $\mathrm{r}^{2}=0.976$. Relevant to the objective of the study,

348 NLC proved to be a suitable carrier of citral as it provides a slow release of it in time dependent

349 manner. A biphasic drug release pattern was observed in which a drug burst release was

350 observed at the initial stage and followed by sustained release at a constant rate later on. This

351 phenomenon might be explained due to the lipid imperfect crystalline structured of the NLC to

352 allow high drug loaded in the system (Müller et al., 2002). Subsequently, this also affects the

353 release of the drug as it becomes loose and causing high rate of the drug released (Uprit et

354 al.,2013). In addition, as the size of the nanoparticle is getting smaller, the specific surface area

355 of nanoparticle is increased and thus the drug release became faster (Moghimi, et al., 2005).

356 Therefore, the robust release rate in the initial stage of this in-vitro drug release study was

357 resulted from these two consequences. Consequently, citral in NLC-citral showed slower release

358 profile. Previous study has confirmed that the slow release of the drug was dependent on the

359 entrapment efficiency and homogenous entrapment of the drug throughout the system (Moghimi

360 et al., 2005). 


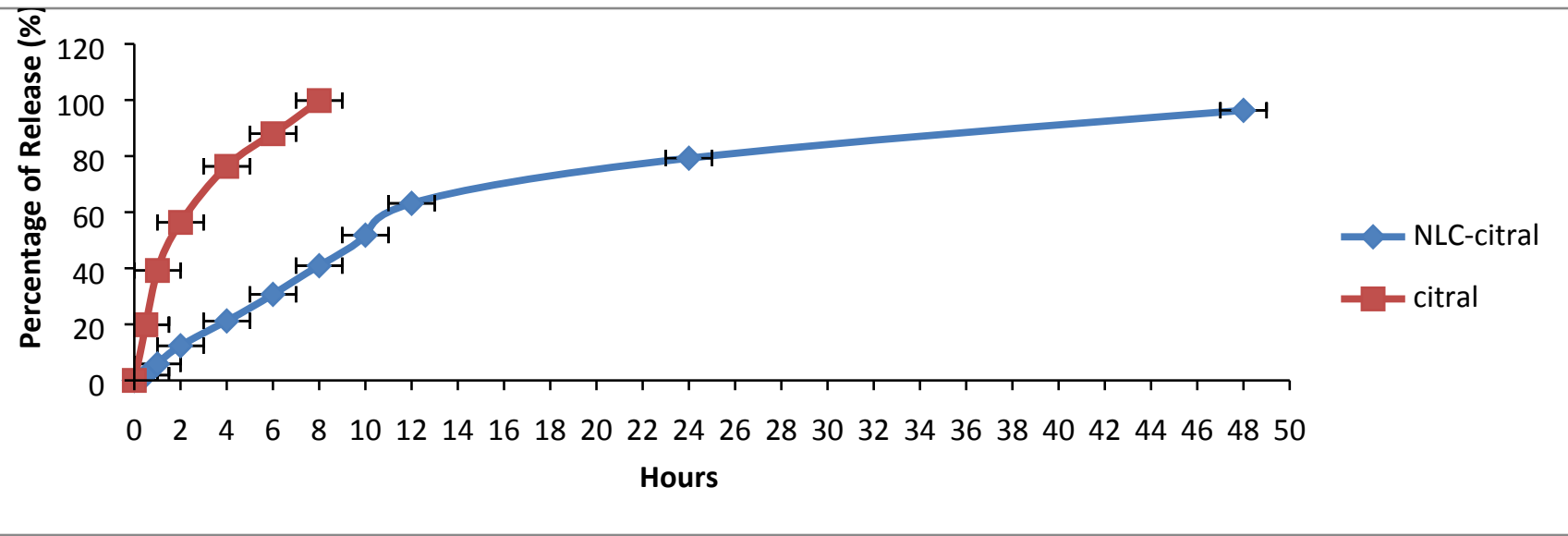

362 Figure 2: The in-vitro drug release study of NLC-citral and citral (control). Each point 363 represents mean and standard deviation $(\mathbf{n}=3)$.

\section{In vitro splenocytes viability (MTT)}

366 To examine the effect of splenocytes proliferation after being treated with NLC-Citral

367 formulation, the MTT assay was conducted. The in vitro proliferation response of splenocytes

368 treated with NLC-citral and citral was investigated and showed in Figure 3. There was no

369 significant decline in the viability of splenocytes detected from treatment with NLC-citral (99.8

$370 \pm 1.7 \%)$ at the highest concentration given to the mice $(30 \mu \mathrm{g} / \mathrm{ml})$ after 24 hours post-treatment

371 as compared to NLC-Blank $(99.5 \pm 0.98 \%)$ which suggests that the formulation did not induce

372 toxicity towards the proliferation of mice splenocytes after being treated (Chen et al., 2009).

373 According to Figure 3, the percentage of splenocytes viability did not change in the treatment of

374 NLC-citral at any concentration given. However, in the citral treated group there was a decrease

375 in splenocytes viability from $99.7 \%$ to $95.2 \%$ at the concentration of $15 \mu \mathrm{g} / \mathrm{mL}$ and $30 \mu \mathrm{g} / \mathrm{mL}$

376 respectively. 
377 It was demonstrated that the NLC-Citral with several concentrations did not alter the effects of

378 splenocytes proliferation treated. As a whole, NLC-Citral has the beneficial effect on immune-

379 stimulation as the splenocytes proliferation was more noticeably better for NLC-citral treated

380 than citral treated group. Immune cells such as $\mathrm{T}$ and $\mathrm{B}$ cells play an important role in the host

381 defense system. Splenocytes proliferation indicates the ability to increase numbers of immune

382 markers which could stimulate the host immunity (Rescigno et al., 2007).

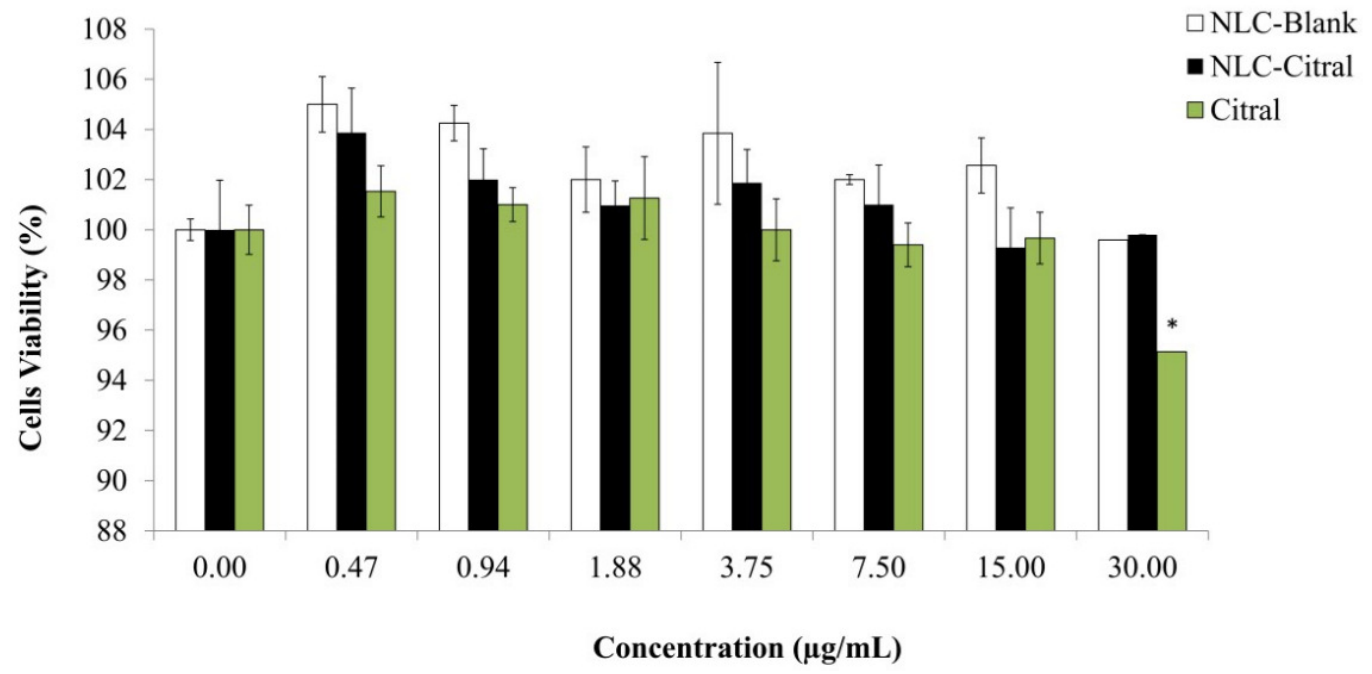

384

385 Figure 3: The percentage of splenocytes viability after being treated with various 386 concentrations of NLC-citral and pure citral ranging from $0.47 \mu \mathrm{g} / \mathrm{mL}$ to $30 \mu \mathrm{g} / \mathrm{mL}$ for 24 387 hours. Each point is represent the mean \pm standard deviation $(n=3)$. Significance was set at 388 p $<0.05$ comparing between groups with $(* *)$ to NLC-Blank and (*) to citral.

In vivo Toxicity Study

All mice treated with NLC-citral and citral survived till the end of the 28 days of treatment.

391 According to Table 2, there were no mortality, body weight changes, and conspicuous toxic

392 signs developed in treated mice throughout the study time. For biochemical analysis, there was a non-significant increase in the serum ALT level of NLC-citral treated group as compared to 
394 NLC-Blank. Meanwhile, the citral treated group showed a decrease in ALT value. Besides,

395 creatinine level of the NLC-citral and citral treated groups has decreased than NLC-Blank.

396 This observation indicates that there was no overt toxicity occurred in NLC-citral and citral

397 treated mice. Even though slight changes were observed in the level of markers from the

398 biochemistry results, there was no significant modification detected. Hepatic damage is a serious

399 issue that should be taken into consideration in the new formulation of compound or drugs.

400 Additional AST enzyme will be released into the bloodstream when liver is damaged that

401 indicates a hepatic injury in the host (Sharma \& Shukla, 2011). ALT level is a main indicator in

402 the detection of liver damage by drug or hepatotoxins in the bodies (Shirodkar et al., 2015).

403 Eventually, the increase is AST level caused an elevation in the level of ALT (Giannini et 404 al.,2005). Moreover, the abnormal levels of ALP, AST and ALT in the serum most often indicate 405 a problem with the liver, gall bladder and heart (Shimizu, 2008). In addition, creatinine level was 406 assessed to view the renal function, an elevation of the creatinine level in the blood signifies

407 impaired kidney function or kidney disease (Shlipak et al., 2013). There were no significant 408 alterations in the levels of kidney and renal biochemical detector molecules in the NLC-Citral 409 and Citral treated groups of mice. Pharmacology study has claimed that Citral is not toxic for

410 human consumption as indicated by no changes and abnormalities in serum glucose, urea, 411 proteins and creatinine level in the urine analysis (Carlini et al., 1986). 
417 Table 2: The observation of mortality, body weight changes, toxicity signs and serum 418 biochemical analysis of NLC-Blank, NLC-Citral and Citral treated groups. Values 419 represent means and standard deviation.

\begin{tabular}{|c|c|c|c|c|c|c|c|}
\hline Group & $\begin{array}{l}\text { Mortality } \\
\text { at } 28^{\text {th }} \text { days }\end{array}$ & $\begin{array}{c}\text { Body weight } \\
\text { Changes }\end{array}$ & $\begin{array}{l}\text { Toxic } \\
\text { Signs }\end{array}$ & $\begin{array}{c}\text { ALT } \\
(\mathrm{U} / \mathrm{L})\end{array}$ & $\begin{array}{l}\text { ALP } \\
(\mathrm{U} / \mathrm{L})\end{array}$ & $\begin{array}{c}\text { AST } \\
(\mathrm{U} / \mathrm{L})\end{array}$ & $\begin{array}{c}\text { CREATININ } \\
(\mu \mathrm{mol} / \mathrm{L})\end{array}$ \\
\hline $\begin{array}{c}\text { NLC-Blank } \\
\text { (Control) }\end{array}$ & NONE & NONE & NONE & $\begin{array}{c}75.2 \pm \\
0.37\end{array}$ & $\begin{array}{l}230 \pm \\
0.65\end{array}$ & $\begin{array}{c}348.2 \pm \\
1.56\end{array}$ & $\begin{array}{l}49.0 \pm \\
1.09\end{array}$ \\
\hline $\begin{array}{c}\text { NLC- } \\
\text { CITRAL }\end{array}$ & NONE & NONE & NONE & $\begin{array}{c}76.1 \pm \\
1.03\end{array}$ & $\begin{array}{c}225 \pm \\
0.98\end{array}$ & $\begin{array}{c}351.9 \pm \\
0.98\end{array}$ & $\begin{array}{c}47.0 \pm \\
0.97\end{array}$ \\
\hline CITRAL & NONE & NONE & NONE & $\begin{array}{c}71.5 \pm \\
0.76\end{array}$ & $\begin{array}{l}232 \pm \\
1.34\end{array}$ & $\begin{array}{c}359.0 \pm \\
0.56\end{array}$ & $\begin{array}{c}47.6 \pm \\
1.0\end{array}$ \\
\hline
\end{tabular}

423 NO level from the NLC-citral and citral treated groups was evaluated to study the anti424 inflammatory effects of the formulations. As illustrated in Figure 4, both NLC-citral and citral 425 have significantly reduced the level of NO. The level of nitric oxide in the NLC-citral group was 426 declined from $101 \pm 3.02 \mu \mathrm{M} / \mathrm{mg}$ (NLC-Blank) to $40.91 \pm 3.02 \mu \mathrm{M} / \mathrm{mg}$ of protein while in citral 427 the value was dropped to $46.16 \pm 4.02 \mu \mathrm{M} / \mathrm{mg}$ of protein.

428 Nitric Oxide (NO) is a signaling molecule that initiates inflammation under normal conditions.

429 High concentrations of NO in the immune system was relatively regulated by cytokine-activated 
430 macrophage(Sharma et al., 2007). The level of NO in the NLC-citral treated spleen has

431 significantly reduced as compared to the NLC-Blank. Chronic inflammation facilitates cancer-

432 related potentials such as inhibition of cell death programs, induce angiogenesis and assists

433 metastasis (Multhoff, Molls, \& Radons, 2011). Hence, the reduction NO level in NLC-citral

434 depicts the anti-inflammatory effect possessed in the formulation.

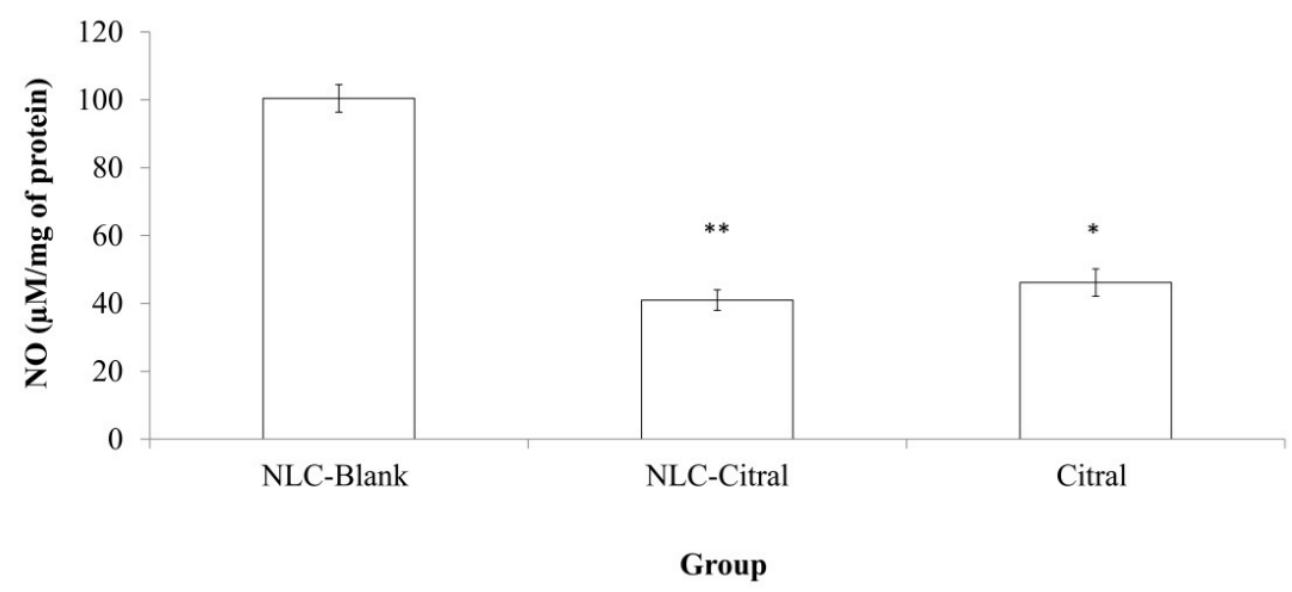

436 Figure 4: Bar chart analysis of the level of Nitric Oxide (NO) detected in the mice 437 splenocytes harvested from the NLC-Blank, NLC-citral and citral treated groups after 28 438 days of treatment $(50 \mathrm{mg} / \mathrm{kg})$. Each value represents the means \pm standard deviation. 439 Significance was set at $\mathbf{p}<\mathbf{0 . 0 5}$ comparing between groups with (*) to NLC-Blank and (**) 440 to citral.

\section{Splenocytes immunophenotyping}

443 To determine the immune response affected from the NLC-citral and citral treatment as

444 compared to the NLC-Blank, in vivo immunophenotyping assay was done. Three different 445 conjugated staining of CD3 (FITC), CD4 (PE) and CD8 (APC) T-cells immune markers were 446 selected to stain the harvested splenocytes from all groups. As shown in Figure 5, the percentage 447 of CD4/CD3 T-cell population has significantly increased in the NLC-citral and citral treated 
448 groups as compared to the NLC-Blank. The level of CD4/CD3 T-cells population increased from

$44913.97 \pm 0.19 \%$ in NLC-Blank to $20.12 \pm 0.40 \%$ in the NLC-citral treated splenocytes and $18.44 \pm$ $450 \quad 0.50 \%$ in the citral treated splenocytes $(50 \mathrm{mg} / \mathrm{kg})$. Furthermore, the level of CD8/CD3 T-cell 451 population was also increased, similar to the pattern of elevation observed in the CD3/CD4 452 population. It can be observed from Figure 5 that the percentage of CD8/CD3 T-cells population 453 in the NLC-citral and citral treated splenocytes has significantly increased to $9.56 \pm 0.32 \%$ and $4548.82 \pm 0.16 \%$ respectively.

455 NLC-citral has increased the percentage of T-cells population higher than citral for all three T456 cells immune markers as compared to the NLC-Blank within the splenocytes population.

457 Previous study reported that citral exhibited potential as an immunomodulatory agent in murine 458 macrophage (Bachiega \& Sforcin, 2011). Therefore, the increase of CD4/CD3 and CD8/CD3 in 459 both NLC-citral and citral treated splenocytes conveyed that these treatments have induced the 460 immunomodulatory effect of the mice. Studies on the immune responses have continued to gain 461 significant attraction due to the importance of immune system in combating against common 462 critical diseases such as cancer (Dunkelberger \& Song, 2010). T-cell immune response 463 impairments and dysfunction almost appear in many chronic diseases such as cancer and Human 464 immunodeficiency virus (HIV) infection disease (Kinter et al., 2004). CD4/CD3 is the T-helper 465 lymphocyte cells while CD8/CD3 is the cytotoxic T cells (Anderson et al.,1988). The depletion 466 of T-helper cells creates an immune hyper activation conditioned which is usually associated 467 with the immune response deficiency (Février et al., 2011). In addition, a cytotoxic $\mathrm{T}$ 468 lymphocyte cell CD8/CD3 leads to the death of target cells by apoptosis in host defense against 469 various pathogens (Janeway et al.,2001b). Much attention has focused on the role of CD8T-cells 
470 in immunotherapy of cancer as it is capable to destroy tumor cells in in vitro and in vivo studies

471 (Finn, 2003).

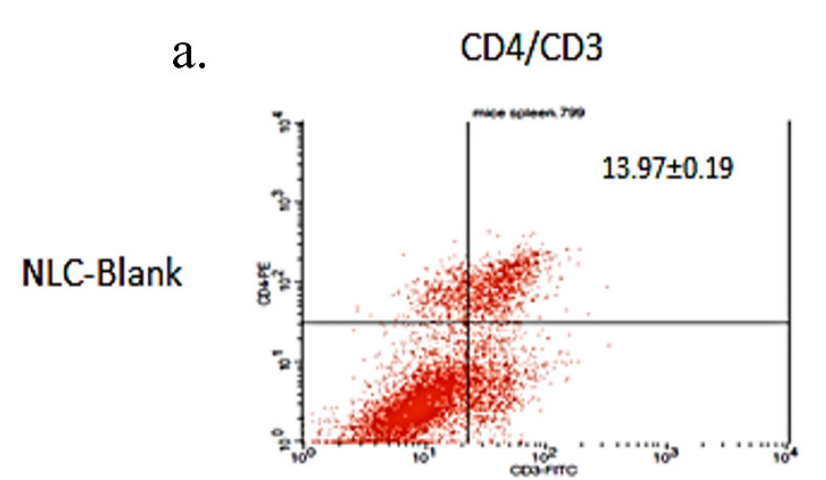

d. $\mathrm{CD} 8 / \mathrm{CD} 3$
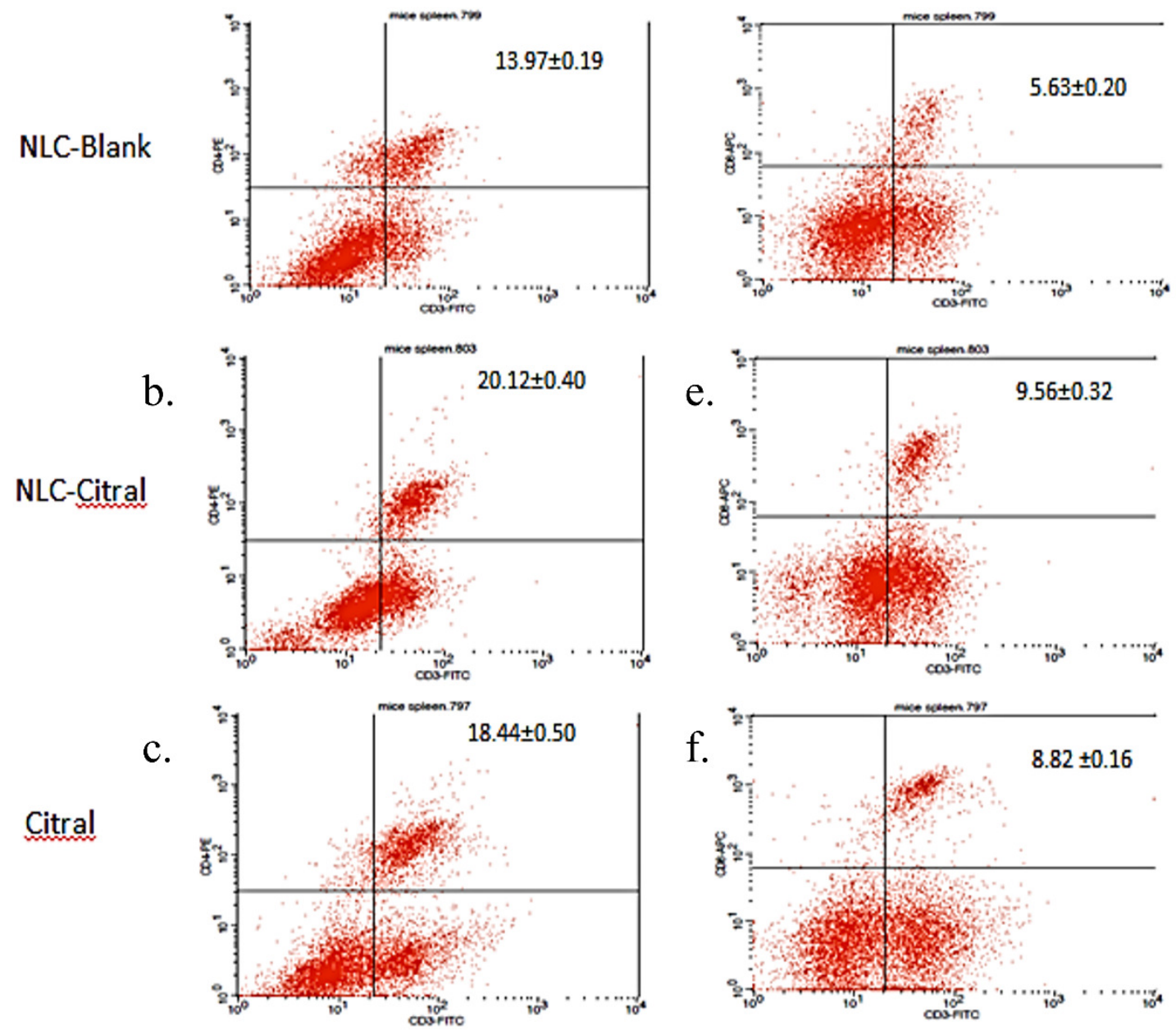

472

473 Figure 5: The histogram analysis of (a-c) $\mathrm{CD3}^{+} \mathrm{CD4}^{+}$and (d-f) $\mathrm{CD3}^{+} \mathrm{CD8}^{+} \mathbf{T}$ lymphocytes in 474 the splenocytes harvested after 28 days of treatment with $50 \mathrm{mg} / \mathrm{kg}$ of NLC-Blank, NLC475 citral and citral. Each value represents mean \pm std with $\mathbf{n}=\mathbf{5}$ mice per group 


\section{Conclusion}

478 In the present study, the formulation of nanostructured lipid carrier encapsulated with citral 479 (NLC-citral) was characterized. The formulation was confirmed to be stable and fell within the 480 nano size range. This was proved based on the results from zeta potential, zeta sizer, 481 polydispersity index and TEM analysis of the nanoparticle. Furthermore, NLC-citral formulation 482 imposes a slow release profile of citral and has enhanced the solubility of the pure citral in the 483 water. Additionally, there was no aberrant toxic sign detected in the blood biochemistry analysis, 484 flow-cytometry immunophenotyping assay, lipid peroxidation (NO) and splenocytes viability 485 between the NLC-Blank (control) and NLC-citral and citral treated mice in the toxicity study 486 conducted. The physicochemical properties have validated that NLC-citral is suitable as a 487 potential delivery system of citral and non-toxic towards healthy cells. Hence, the NLC-citral 488 formulation can be further investigated to confirm its potential as a new delivery system for citral 489 in the treatment of cancer as citral is known to have anti-cancer properties.

\section{References}

Abdelwahab, S. I., Sheikh, B. Y., Taha, M. M. E., How, C. W., Abdullah, R., Yagoub, U., ... Eid, E. E. M. (2013). Thymoquinone-loaded nanostructured lipid carriers: preparation, gastroprotection, in vitro toxicity, and pharmacokinetic properties after extravascular administration. International Journal of Nanomedicine, 8, 2163-72. http://doi.org/10.2147/IJN.S44108

Abu, N., Mohamed, N. E., Tangarajoo, N., Yeap, S. K., Akhtar, M. N., Abdullah, M. P., ... Alitheen, N. B. (2015). In vitro Toxicity and in vivo Immunomodulatory Effects of Flavokawain A and 
499

500

501

502

503

504

505

506

507

508

509

510

511

512

513

514

515

516

Almeida, H., Amaral, M. H., Lobão, P., Silva, A. C., \& Sousa Lobo, J. M. (2014). Applications of Polymeric and Lipid Nanoparticles in Ophthalmic Pharmaceutical Formulations: Present and Future Considerations. J Pharm Pharm Sci (www.cspsCanada.org), 17(3), 278-293.

Andalib, S., Varshosaz, J., Hassanzadeh, F., \& Sadeghi, H. (2012). Optimization of LDL targeted nanostructured lipid carriers of 5-FU by a full factorial design. Advanced Biomedical Research, 1, 45. http://doi.org/10.4103/2277-9175.100147

Anderson, P., Blue, M. L., \& Schlossman, S. F. (1988). Comodulation of CD3 and CD4. Evidence for a specific association between CD4 and approximately 5\% of the CD3:T cell receptor complexes on helper T lymphocytes. Journal of Immunology (Baltimore, Md. : 1950), 140(6), 1732-7.

Bachiega, T. F., \& Sforcin, J. M. (2011). Lemongrass and citral effect on cytokines production by murine macrophages. Journal of Ethnopharmacology, 137(1), 909-913. http://doi.org/10.1016/j.jep.2011.07.021

Carlini, E. A., Contar J de, D. P., Silva-Filho, A. R., da Silveira-Filho, N. G., Frochtengarten, M. L., \& Bueno, O. F. (1986). Pharmacology of lemongrass (Cymbopogon citratus Stapf). I. Effects of teas prepared from the leaves on laboratory animals. Journal of Ethnopharmacology, 17(1), 37-64.

Charles A Janeway, J., Travers, P., Walport, M., \& Shlomchik, M. J. (2001). T cell-mediated cytotoxicity. Garland Science.

Chen, C., Johnston, T. D., Jeon, H., Gedaly, R., McHugh, P. P., Burke, T. G., \& Ranjan, D. (2009). An in 
517 vitro study of liposomal curcumin: stability, toxicity and biological activity in human lymphocytes 518 and Epstein-Barr virus-transformed human B-cells. International Journal of Pharmaceutics, 366(1519 2), 133-9. http://doi.org/10.1016/j.ijpharm.2008.09.009

520 Cragg, G. M., \& Newman, D. J. (2005). Plants as a source of anti-cancer agents. Journal of 521 Ethnopharmacology, 100(1-2), 72-9. http://doi.org/10.1016/j.jep.2005.05.011

522 Dunkelberger, J. R., \& Song, W.-C. (2010). Complement and its role in innate and adaptive immune 523 responses. Cell Research, 20(1), 34-50. http://doi.org/10.1038/cr.2009.139

524 Fang, C., Shi, B., Pei, Y.-Y., Hong, M.-H., Wu, J., \& Chen, H.-Z. (2006). In vivo tumor targeting of 525 tumor necrosis factor- $\alpha$-loaded stealth nanoparticles: Effect of MePEG molecular weight and particle size. European Journal of Pharmaceutical Sciences, 27(1), 27-36. http://doi.org/10.1016/j.ejps.2005.08.002

Février, M., Dorgham, K., \& Rebollo, A. (2011). CD4+ T Cell Depletion in Human Immunodeficiency 529 Virus (HIV) Infection: Role of Apoptosis. Viruses, 3(12), 586-612. http://doi.org/10.3390/v3050586

Finn, O. J. (2003). Cancer vaccines: between the idea and the reality. Nature Reviews. Immunology, 3(8), 630-41. http://doi.org/10.1038/nri1150

532 Foster, K. A., Yazdanian, M., \& Audus, K. L. (2001). Microparticulate uptake mechanisms of in-vitro cell 533 culture models of the respiratory epithelium. Journal of Pharmacy and Pharmacology, 53(1), 57534 66. http://doi.org/10.1211/0022357011775190

535 Ghosh, K. (2013). Anticancer effect of lemongrass oil and citral on cervical cancer cell lines, 3(4), 41-48. 
537 Giannini, E. G., Testa, R., \& Savarino, V. (2005). Liver enzyme alteration: a guide for clinicians. $C M A J$ : Canadian Medical Association Journal = Journal de l'Association Medicale Canadienne, 172(3), 367-79. http://doi.org/10.1503/cmaj.1040752

Honary, S., \& Zahir, F. (2013). Effect of Zeta Potential on the Properties of Nano-Drug Delivery Systems - A Review (Part 1). Tropical Journal of Pharmaceutical Research, 12(2), 255-264. http://doi.org/10.4314/tjpr.v12i2.19

How, C. W., Rasedee, A., Manickam, S., \& Rosli, R. (2013). Tamoxifen-loaded nanostructured lipid carrier as a drug delivery system: characterization, stability assessment and cytotoxicity. Colloids and Surfaces. B, Biointerfaces, 112, 393-9. http://doi.org/10.1016/j.colsurfb.2013.08.009 of citral on NO production by suppression of iNOS expression and NF-kappa B activation in RAW264.7 cells. Archives of Pharmacal Research, 31(3), 342-9. http://doi.org/10.1007/s12272-

Kasongo, K. W., Pardeike, J., Müller, R. H., \& Walker, R. B. (2011). Selection and characterization of suitable lipid excipients for use in the manufacture of didanosine-loaded solid lipid nanoparticles and nanostructured lipid carriers. Journal of Pharmaceutical Sciences, 100(12), 5185-96. http://doi.org/10.1002/jps.22711

554 Kinter, A. L., Hennessey, M., Bell, A., Kern, S., Lin, Y., Daucher, M., ... Fauci, A. S. (2004). $\mathrm{CD} 25(+) \mathrm{CD} 4(+)$ regulatory $\mathrm{T}$ cells from the peripheral blood of asymptomatic HIV-infected 
556 individuals regulate $\mathrm{CD} 4(+)$ and $\mathrm{CD} 8(+)$ HIV-specific $\mathrm{T}$ cell immune responses in vitro and are 557 associated with favorable clinical markers of disease status. The Journal of Experimental Medicine, 558 200(3), 331-43. http://doi.org/10.1084/jem.20032069

559 Lee, M.-K., Lim, S.-J., \& Kim, C.-K. (2007). Preparation, characterization and in vitro cytotoxicity of 560 paclitaxel-loaded sterically stabilized solid lipid nanoparticles. Biomaterials, 28(12), 2137-46. $561 \quad$ http://doi.org/10.1016/j.biomaterials.2007.01.014

562 Luan, J., Zhang, D., Hao, L., Li, C., Qi, L., Guo, H., ... Zhang, Q. (2013). Design and characterization of 563 Amoitone B-loaded nanostructured lipid carriers for controlled drug release. Drug Delivery, 20(8), 564 324-30. http://doi.org/10.3109/10717544.2013.835007

565 Mitra, S., Gaur, U., Ghosh, P. C., \& Maitra, A. N. (2001). Tumour targeted delivery of encapsulated 566 dextran-doxorubicin conjugate using chitosan nanoparticles as carrier. Journal of Controlled 567 Release: Official Journal of the Controlled Release Society, 74(1-3), 317-23.

568 Moghimi, S. M., Hunter, A. C., \& Murray, J. C. (2005). Nanomedicine: current status and future 569 prospects. FASEB Journal: Official Publication of the Federation of American Societies for Experimental Biology, 19(3), 311-30. http://doi.org/10.1096/fj.04-2747rev

571 Mohamad, N. E., Abu, N., Rahman, H. S., Ky, H., Ho, W. Y., Lim, K. L., .. Yeap, S. K. (2015). 572 Nanostructured lipid carrier improved in vivo anti-tumor and immunomodulatory effect of 573 Zerumbone in 4T1 challenged mice. RSC Adv., 5(28), 22066-22074. 574 http://doi.org/10.1039/C5RA00144G

575 Montenegro, L., Campisi, A., Sarpietro, M. G., Carbone, C., Acquaviva, R., Raciti, G., \& Puglisi, G. 
576 (2011). In vitro evaluation of idebenone-loaded solid lipid nanoparticles for drug delivery to the

577 brain. Drug Development and Industrial Pharmacy, 37(6), 737-746.

$578 \quad$ http://doi.org/10.3109/03639045.2010.539231

579 Mudshinge, S. R., Deore, A. B., Patil, S., \& Bhalgat, C. M. (2011). Nanoparticles: Emerging carriers for 580 drug delivery. Saudi Pharmaceutical Journal: SPJ: The Official Publication of the Saudi 581 Pharmaceutical Society, 19(3), 129-41. http://doi.org/10.1016/j.jsps.2011.04.001

582 Mukherjee, B., Santra, K., Pattnaik, G., \& Ghosh, S. (2008). Preparation, characterization and in-vitro 583 evaluation of sustained release protein-loaded nanoparticles based on biodegradable polymers. $584 \quad$ International Journal of Nanomedicine, 3(4), 487-96.

585 Müller, R. ., Radtke, M., \& Wissing, S. . (2002). Nanostructured lipid matrices for improved 586 microencapsulation of drugs. International Journal of Pharmaceutics, 242(1-2), 121-128. $587 \quad$ http://doi.org/10.1016/S0378-5173(02)00180-1

588 Multhoff, G., Molls, M., \& Radons, J. (2011). Chronic inflammation in cancer development. Frontiers in 589 Immunology, 2, 98. http://doi.org/10.3389/fimmu.2011.00098

590 Pardeshi, C., Rajput, P., Belgamwar, V., Tekade, A., Patil, G., Chaudhary, K., \& Sonje, A. (2012). Solid 591 lipid based nanocarriers: an overview. Acta Pharmaceutica (Zagreb, Croatia), 62(4), 433-72. $592 \quad$ http://doi.org/10.2478/v10007-012-0040-z

593 Patel, D., Dasgupta, S., Dey, S., Ramani, Y. R., Ray, S., \& Mazumder, B. (2012). Nanostructured Lipid 594 Carriers (NLC)-Based Gel for the Topical Delivery of Aceclofenac: Preparation, Characterization, 595 and In Vivo Evaluation. Scientia Pharmaceutica, 80(3), 749-64. 
597

598

599

600

601 602 603

Rahman, H. S., Rasedee, A., How, C. W., Abdul, A. B., Zeenathul, N. A., Othman, H. H., ... Yeap, S. K. (2013). Zerumbone-loaded nanostructured lipid carriers: preparation, characterization, and antileukemic effect. International Journal of Nanomedicine, 8(1), 2769-81. http://doi.org/10.2147/IJN.S45313

Rahman, H. S., Rasedee, A., Othman, H. H., Chartrand, M. S., Namvar, F., Yeap, S. K., .. How, C. W. (2014). Acute toxicity study of zerumbone-loaded nanostructured lipid carrier on BALB/c mice model. BioMed Research International, 2014(3), 563930. http://doi.org/10.1155/2014/563930

Rescigno, M., Avogadri, F., \& Curigliano, G. (2007). Challenges and prospects of immunotherapy as cancer treatment. Biochimica et Biophysica Acta, 1776(1), 108-23. http://doi.org/10.1016/j.bbcan.2007.07.003

Ress, N. B., Hailey, J. R., Maronpot, R. R., Bucher, J. R., Travlos, G. S., Haseman, J. K., ... Hejtmancik, M. R. (2003). Toxicology and carcinogenesis studies of microencapsulated citral in rats and mice. Toxicological Sciences : An Official Journal of the Society of Toxicology, 71(2), 198-206.

Salerno, C., Carlucci, A. M., \& Bregni, C. (2010). Study of in vitro drug release and percutaneous absorption of fluconazole from topical dosage forms. AAPS PharmSciTech, 11(2), 986-93. http://doi.org/10.1208/s12249-010-9457-1

Seki, J., Sonoke, S., Saheki, A., Fukui, H., Sasaki, H., \& Mayumi, T. (2004). A nanometer lipid emulsion, lipid nano-sphere (LNS), as a parenteral drug carrier for passive drug targeting. International Journal of Pharmaceutics, 273(1-2), 75-83. http://doi.org/10.1016/j.ijpharm.2003.12.022 
616 Sharma, D., Chelvi, T. P., Kaur, J., Chakravorty, K., De, T. K., Maitra, A., \& Ralhan, R. (1996). Novel

617 Taxol formulation: polyvinylpyrrolidone nanoparticle-encapsulated Taxol for drug delivery in 618 cancer therapy. Oncology Research, 8(7-8), 281-6.

619 Sharma, J. N., Al-Omran, \& Parvathy, S. (2007). Role of nitric oxide in inflammatory diseases. 620 Inflammopharmacology, 15(6), 252-9. http://doi.org/10.1007/s10787-007-0013-x

621 Sharma, N., Madan, P., \& Lin, S. (2015). Effect of process and formulation variables on the preparation 622 of parenteral paclitaxel-loaded biodegradable polymeric nanoparticles: A co-surfactant study. Asian 623 Journal of Pharmaceutical Sciences. http://doi.org/10.1016/j.ajps.2015.09.004

624 Sharma, N., \& Shukla, S. (2011). Hepatoprotective potential of aqueous extract of Butea monosperma 625 against $\mathrm{CCl}(4)$ induced damage in rats. Experimental and Toxicologic Pathology: Official Journal of the Gesellschaft Für Toxikologische Pathologie, 63(7-8), 671-6. http://doi.org/10.1016/j.etp.2010.05.009

Shimizu, Y. (2008). Liver in systemic disease. World Journal of Gastroenterology, 14(26), 4111-9.

Shirodkar, R., Misra, C., Gh, C., Shetty, P., Attari, Z., Mutalik, S., \& Lewis, S. (2015). Subacute Toxicity 630 Profile of Lacidipine Nanoformulation in Wistar Rats. TheScientificWorldJournal, 2015, 947623. http://doi.org/10.1155/2015/947623

632 Shlipak, M. G., Matsushita, K., Ärnlöv, J., Inker, L. A., Katz, R., Polkinghorne, K. R., ... Gansevoort, R. 633 T. (2013). Cystatin C versus Creatinine in Determining Risk Based on Kidney Function.

634 Thatipamula, R., Palem, C., Gannu, R., Mudragada, S., \& Yamsani, M. (2011). Formulation and in vitro 
635 characterization of domperidone loaded solid lipid nanoparticles and nanostructured lipid carriers. 636 Daru : Journal of Faculty of Pharmacy, Tehran University of Medical Sciences, 19(1), 23-32.

637 Tiwari, G., Tiwari, R., Sriwastawa, B., Bhati, L., Pandey, S., Pandey, P., \& Bannerjee, S. K. (2012). Drug 638 delivery systems: An updated review. International Journal of Pharmaceutical Investigation, 2(1), 639 2-11. http://doi.org/10.4103/2230-973X.96920

640 Uprit, S., Kumar Sahu, R., Roy, A., \& Pare, A. (2013). Preparation and characterization of minoxidil 641 loaded nanostructured lipid carrier gel for effective treatment of alopecia. Saudi Pharmaceutical 642 Journal: SPJ: The Official Publication of the Saudi Pharmaceutical Society, 21(4), 379-85. 643 http://doi.org/10.1016/j.jsps.2012.11.005

644 Weerawatanakorn, M., Wu, J.-C., Pan, M.-H., \& Ho, C.-T. (2015). Reactivity and stability of selected 645 flavor compounds. Journal of Food and Drug Analysis, 23(2), 176-190. 646 http://doi.org/10.1016/j.jfda.2015.02.001

647

648

649

650

\section{List of abbreviation}

ALP

ALT
Alkaline phosphatase

Alanine aminotransferase 


\begin{tabular}{|c|c|}
\hline APC & Antigen-presenting cells \\
\hline AST & Aspartate Aminotransferase \\
\hline ATCC & Animal Tissue Culture Collection \\
\hline $\mathrm{CDK}$ & Cyclin Dependent Kinase \\
\hline DL & Drug Loading \\
\hline DMEM & Dulbecco's Modified Eagle Medium \\
\hline DMSO & Dimethyl sulfoxide \\
\hline EDTA & Ethylenediaminetetraacetic acid \\
\hline $\mathrm{EE}$ & Entrapment Efficiency \\
\hline FACS & Fluorescence-activated cell sorter \\
\hline HPO & Hydrogenated Palm Oil \\
\hline MTT & 3-(4,5-dimethylthiazol-2-yl)-2,5-diphenyltetrazolium bromide \\
\hline $\mathrm{NaCl}$ & Sodium chloride \\
\hline NO & Nitric oxide \\
\hline PBS & Phosphate buffer saline \\
\hline PI & Propidium Iodide \\
\hline TEM & Transmission Electron Microscope \\
\hline WHO & World Health Organization \\
\hline
\end{tabular}

651

652 